\title{
La sodomie masculine dans les pamphlets révolutionnaires
}

Masculine sodomy in revolutionary pamphlets

\section{Thierry Pastorello}

\section{(2) OpenEdition Journals}

Édition électronique

URL : https://journals.openedition.org/ahrf/11698

DOI : $10.4000 / a h r f .11698$

ISSN : 1952-403X

Éditeur :

Armand Colin, Société des études robespierristes

Édition imprimée

Date de publication : 1 septembre 2010

Pagination : $91-130$

ISBN : 978-2-200-92633-5

ISSN : 0003-4436

Référence électronique

Thierry Pastorello, « La sodomie masculine dans les pamphlets révolutionnaires », Annales historiques de la Révolution française [En ligne], 361 | juillet-septembre 2010, mis en ligne le 01 septembre 2013, consulté le 22 avril 2022. URL : http://journals.openedition.org/ahrf/11698 ; DOI : https://doi.org/ 10.4000/ahrf.11698 


\title{
LA SODOMIE MASCULINE DANS LES PAMPHLETS RÉVOLUTIONNAIRES
}

\author{
Thierry PASTORELLO
}

\begin{abstract}
Une utilisation régulière de la sodomie masculine est décelable dans les pamphlets révolutionnaires. Cette préoccupation doit être replacée dans le cadre des débats sur la sodomie masculine dans la littérature $\mathrm{au} \mathrm{xVIII}{ }^{\mathrm{e}}$ siècle et notamment la littérature libertine. À partir de cette constatation, on observe trois thématiques : la sodomie masculine est utilisée politiquement pour déconsidérer des anciennes élites, mais pas uniquement, afin de les faire paraître dépravées. Elle est définie comme un plaisir antinaturel et hédoniste par essence. On oppose ce plaisir à celui trouvé avec une femme, qui est utile socialement et semble définir le bon patriote. Enfin, la sodomie est aussi assimilée à des facteurs de désordres : formes de prostitution, débauche de la jeunesse, maladies vénériennes.
\end{abstract}

Mots-clés : sodomie, pamphlets révolutionnaires, homosexualité masculine, France.

Une longue tradition française de pamphlets et autres écrits satiriques témoigne du recours au sexe comme arme de critique et de caricature, depuis l'érotisme goliardique au XIII ${ }^{\mathrm{e}}$ siècle (qui exprime dans des termes crus l'obsession sexuelle du passage à l'acte), en passant par les poésies libres et satiriques au début du $\mathrm{XVII}^{\mathrm{e}}$ siècle $^{1}$, sans oublier les mazarinades qui sont d'une particulière crudité. En partie héritiers de cette tradition, les pamphlets révolutionnaires doivent être replacés dans les débats discernables du domaine littéraire et philosophique sur

(1) Didier Foucault, Histoire du libertinage: des goliards au marquis de Sade, Paris, Perrin, 2007, p. 17, 20, 235 et 238. 
les plaisirs sexuels et sur la sodomie en particulier. Au XVIII ${ }^{\mathrm{e}}$ siècle, un débat émerge en effet sur la sodomie au sens de pratiques sexuelles entre hommes. Ces discours peuvent être perçus comme un processus de mise à nu des plaisirs. Il y a questionnement sur les différentes facettes des plaisirs vénériens, comme le souligne Alain Corbin ${ }^{2}$. Cette liberté de parole est porteuse d'ambiguïté, car elle contribue à définir des plaisirs particuliers qui sont du domaine du fortement transgressif. En revanche, on pourrait, à la vue de certains textes pamphlétaires, émettre l'hypothèse d'une volonté de justification ${ }^{3}$. En tout état de cause, il y a bien un début d'identification du sodomite masculin comme personne spécifique dans cette analyse des plaisirs, perceptible aussi bien dans la littérature que dans les écrits licencieux.

La sodomie masculine est abordée de deux façons dans la littérature au cours du XVIII ${ }^{\mathrm{e}}$ siècle. Dans les écrits philosophiques, la sodomie est présentée dans le cadre d'un débat sur les notions de nature et contre nature. La nature est-elle polymorphe comme certains le soutiennent, notamment Denis Diderot? C'est le sens profond de son affirmation : «Tout ce qui est dans la nature ne peut être ni contre-nature, ni hors-nature, je n'en excepte pas même la chasteté et la continence volontaire $\rangle^{4}$. Parallèlement, Jean-Jacques Rousseau replace la sodomie masculine dans sa théorie du sentiment naturel. À ses yeux, le sentiment antinaturel ne peut être que le produit du dévoiement des sociétés ${ }^{5}$. Cette théorie est importante à souligner, car les pamphlets révolutionnaires s'abreuveront d'un rousseauisme sommaire, notamment avec des auteurs que Robert Darnton a nommé les « Rousseaux de ruisseau " ${ }^{6}$. Quant à lui, Montesquieu cherche les causes de la sodomie masculine dans certaines coutumes déplacées. Ainsi, une bonne police et de saines coutumes sont-elles de nature à résoudre le problème. Il souscrit au concept de contre-nature ${ }^{7}$. Voltaire, lui, nie l'affirma-

(2) Alain Corbin, L'harmonie des plaisirs : les manières de jouir du siècle des lumières, à l'avènement de la sexologie, Paris, Perrin, 2007.

(3) Voir notamment le texte L'ombre de Deschauffours datant de 1739 et qui est un plaidoyer en faveur de Benjamin Deschauffours brûlé en place de Grève pour sodomie en 1726 (pour le texte de cette pièce voir Claude Courouve, Les gens de la manchette, Paris, 1978).

(4) Denis Diderot, Mémoires, correspondance et ouvrages inédits, Paris 1830-1831, Tome 4, p. 234.

(5) Voir Pierre ZaOuI, " philosophie », dans Louis Georges Tin, Dictionnaire de l'homophobie, Paris, PUF, 2003, p. 319-320.

(6) Robert DARNTON, Bohème littéraire et révolution : le monde des livres au XVIII siècle, Paris, Gallimard, le Seuil, 1983.

(7) Pour la position de Montesquieu, voir Montesquieu, L'esprit des lois, Paris, Larousse, 1995, livre VI, chapitre XII, Du crime contre la nature. 
tion selon laquelle certaines civilisations aient pu encourager la pratique de ces comportements sexuels. Par ailleurs, il semble expliquer le comportement homosexuel chez les jeunes adolescents n'ayant pas pris pleinement conscience de l'altérité des sexes ${ }^{8}$. Parallèlement, la visibilité de ces pratiques au sein de certaines civilisations anciennes fait débat. Est-ce la marque de l'infériorité de ces civilisations ${ }^{9}$ ? De plus, on assiste à une description archéologique des plaisirs de la chair. On met à nu le désir et on expose la jouissance sexuelle dans toutes ses variantes ${ }^{10}$. Ainsi, la sodomie comme pratique sexuelle est abordée dans des textes littéraires et notamment dans toute une littérature licencieuse. Certains de ces textes furent jugés obscènes, d'autres manient la caricature. Certains textes licencieux, comme Le diable au corps d'Andréa de Nerciat (1739-1800) édité en 1786, furent publiés sous un pseudonyme. On y retrouve des caractéristiques que l'on rencontre dans les pamphlets révolutionnaires, notamment l'utilisation d'une langue très crue avec des termes comme « le con », « le cul», « les conistes», « les culistes » ou « fourgonner les anus », «putains », « ribauds », " goût» et la dénonciation des mœurs libertines du clergé. Robert Darnton a souligné l'existence, à la veille de la Révolution, d'une bohème littéraire. Ces milieux, souligne-t-il, comportaient une bonne dose d'écrivassiers qui prennent quelques facilités pour du talent ${ }^{11}:$ « Ils étaient en guerre contre une organisation de la culture, et une production du livre, régentées par le privilège $»^{12}$. Or, précisément, cette bohème littéraire faisait preuve d'une agressivité doublée de vulgarité, des traits que l'on retrouve aussi dans les pamphlets révolutionnaires ${ }^{13}$. Elle utilisait un sensationnalisme sexuel et elle pourvoyait en ouvrages pornographiques un public intéressé par les débauches supposées de la Cour ${ }^{14}$.

Par ailleurs, il faut, pour comprendre ces pamphlets révolutionnaires, souligner l'extinction progressive du crime de sodomie : sa dernière application pure a lieu en 1750 avec Bruno Lenoir et Jean Diot, surpris en plein Paris par un sergent du guet. Ce processus d'extinction

(8) Voltaire, Dictionnaire philosophique portatif, article amour dit socratique, 1764 (http://www.hs-augsburg.de/ Harsch/gallica/Chronologie/18siecle/Voltaire/vol_dp05.html).

(9) Voir notamment les affirmations de Augustin BARRUEL, Les helviennes ou lettres provinciales philosophiques, Amsterdam, Laporte, 1781 ou celles de Jean BOUHIER, Recherches et dissertations sur Hérodote, Dijon, 1746, p. 211.

(10) Voir Michel Delon, « De la Régence aux Lumières : les milles et unes ressources du désir », Magazine littéraire, no 371 décembre 1998, p. 31.

(11) Robert DaRnton, Bohème littéraire et révolution [...], op. cit., p. 18.

(12) Jacques Solé, La révolution en question, Paris, Éd. du Seuil, 1988, p. 32.

(13) Voir Robert DARNTON, Bohème littéraire et révolution [...], op. cit., p. 27-28.

(14) Jacques Solé, La révolution en question [...], op. cit., p. 33. 
progressive aboutit à la disparition du crime de sodomie en 1791. Or, certains de ces pamphlets s'élèvent contre la tolérance accordée à ces pratiques. Cette impression se fonde sur l'existence d'une subculture sodomite déjà bien présente dans le Paris du XVIII ${ }^{\mathrm{e}}$ siècle. Enfin, lorsque l'on parle de sodomie qu'entend-on au niveau épistémologique? Antérieurement, ce terme désignait toutes les pratiques sexuelles n'aboutissant pas à la procréation : on trouvait dans les procès intentés à des hommes pour crime de sodomie, des hommes accusés de bestialité ${ }^{15}$. Florence Tamagne note à cet égard que "l'accusation de sodomie visait une forme d'acte sexuel et non une catégorie de personnes : ainsi même si du Moyen Âge au XVIII ${ }^{\mathrm{e}}$ siècle des homosexuels en furent victimes, les lois sur la sodomie pouvaient s'appliquer à des relations hétérosexuelles, à la bestialité et même de manière beaucoup plus vague à l'hérésie et à la trahison ${ }^{16}$. Cependant, au XVIII ${ }^{\mathrm{e}}$ siècle, il y a un glissement dans l'emploi du terme de sodomie et sodomite. En effet, ce vocable finit par désigner ce que nous appelons aujourd'hui un homosexuel masculin. Enfin, il est nécessaire de relier ces pamphlets aux événements propres de la Révolution française : certains d'entre eux sont des réactions aux événements de la première partie de la Révolution, comme le texte pamphlétaire nommé Le bordel apostolique, réaction à l'attitude de Pie VI face à la Révolution, à la suite de l'adoption de la Constitution civile du clergé. À partir de ce contexte d'ensemble, il faut noter combien ces écrits satiriques utilisent le thème de la sodomie masculine, mais aussi combien ils véhiculent des images spécifiques sur cette dernière, par opposition à la masculinité. À partir de cette problématique, il convient de discerner de quelle manière ces textes décrivent la sodomie. Globalement, nous distinguons trois thématiques : un usage politique de la sodomie masculine, la sodomie masculine comme plaisir antinaturel et hédoniste par essence, enfin la sodomie masculine comme facteur de désordres. Pour terminer cette introduction, nous pouvons faire référence à une contribution de Patrick Wald Lasowski parue dans Le Magazine littéraire, qui souligne combien la langue érotique est prospère pendant la Révolution et comment elle affirme une virilité révolutionnaire, tout en marquant une volonté de régénérescence ${ }^{17}$.

(15) Voir Fernand Fleuret et Louis Perceau, Les procès de sodomie aux XVI ${ }^{e}$, XVII et XVIII siècles : Publiés d'après les documents judiciaires conservés à la Bibliothèque nationale, Paris, Bibliothèque des curieux, 1920.

(16) Florence TAMAgne, « Homosexualités, le difficile passage de l'analyse des discours à l'étude des pratiques », Histoire \& sociétés, nº 3, 2002, p. 6.

(17) Patrick Wald-Lasowski, « Les fouteries chantantes de la Révolution », Magazine littéraire $\mathrm{n}^{\circ} 426$, décembre 2003, p. 35-38. 


\section{L'usage politique de la sodomie masculine dans les pamphlets révolutionnaires}

L'usage politique de la sodomie masculine a pour but de déconsidérer d'anciennes élites en les accusant de dépravation. On mentionne bien entendu l'ancienne noblesse et le clergé comme adeptes de ces pratiques sexuelles. Leurs prétendues orgies sexuelles sont dénoncées, et ceci est la marque intangible de leur dégénérescence.

« Votre douce charité font espérer au clergé de France que vous lui accordez votre sainte bénédiction, et qu'il sera constamment agréable à votre bienfaisance paternité, d'octroyer à tous les membres qui composent l'Église française, la permission d'ouvrir un bordel public, où ils puissent se réunir et soulager réciproquement leurs désirs antiphysiques $»^{18}$.

Le fait de flétrir le clergé tient à plusieurs causes. D'une part, ces attaques se comprennent par le fait que le clergé est l'un des deux ordres privilégiés de l'Ancien Régime. Robert Darnton souligne combien les libelles expriment un sentiment de mépris total envers une élite corrompue et le message délivré est que la maladie sociale avait sa source au sommet $^{19}$. D'autre part, les causes de ces attaques contre le clergé tiennent autant à la perception de l'homosexualité qu'à la position du clergé. Ce dernier est composé d'hommes célibataires, en raison de l'obligation du célibat des prêtres. Ces hommes vivent entre eux. Or, dès la Révolution on se préoccupe des célibataires. Certains s'en prennent au célibat des prêtres ${ }^{20}$. Ces attaques à l'égard du clergé sont à ranger dans ce que René Rémond nomme un premier type d'anticléricalisme. On reprend la morale chrétienne et on la transforme en morale naturelle. Dès lors, c'est au nom de celle-ci que les clercs sont attaqués. Ainsi, ils n'observeraient pas les préceptes qu'ils sont chargés de professer. Les religieux falsifieraient la vérité et pratiqueraient la duplicité et l'hypocrisie, et on attaque particulièrement les jésuites ${ }^{21}$. Cet anticléricalisme sera exacerbé par les événements accompagnant la Constitution civile du clergé en 1790 puis la condamnation par le pape Pie VI des principes de la Révolution

(18) Bordel apostolique institué par Pie VI, pape, en faveur du clergé de France, Paris, impr. De l'abbé Grossier, 1790, p. 8.

(19) Voir Robert DARNTON, Bohème littéraire et révolution [...], op. cit., p. 31.

(20) Voir Jean Claude Bologne, Histoire du célibat et des célibataires, Paris, Fayard, 2004, p. 199 .

(21) René RÉmOnD, L'anticléricalisme en France de 1815 à nos jours, Bruxelles, Éditions Complexe, 1992, p. 27-29. 
française en mars 1791, et ils peuvent expliquer ces liens entre clergé et sodomie masculine. Ce contexte politique global permet de situer ces libelles contre les mœurs du clergé. Tous ces textes vont dans le sens de la dénonciation de ses mœurs corrompues, et la pratique de la sodomie masculine semble être perçue comme une pratique sexuelle par excellence de ces hommes d'Église. Le Bordel apostolique institué par Pie VI, dont nous avons fait mention, décrit ainsi les prétendues mœurs pédérastes du clergé :

«Il ne serait point question de fouterie naturelle; Qu'on n'y occuperait ses forces et son temps, qu'à soulager les ardeurs de la bougrerie, de la pédérastie et de la bardacherie, que pour interdire toute jouissance toute manipulation des cons, des tétons, les femmes, les filles seraient exclues de ce lieu de délices $»^{22}$.

En même temps, la sodomie masculine est assimilée, dans ce pamphlet, à l'inutilité sociale, car les clercs sont célibataires. Ce sont aussi des hommes qui ne sont pas astreints au travail et qui vivraient de celui d'autrui, ceci expliquant ces représentations ${ }^{23}$. Par opposition, la sexualité naturelle est facteur d'utilité. La sodomie masculine est clairement opposée au plaisir avec la femme, et elle est présentée comme une pratique sexuelle propre au clergé : comme le clergé est parasite, qu'il ne s'occupe que de ses plaisirs et que les clercs sont célibataires, la pratique de la sodomie masculine a toute sa place. On retrouve dans cet écrit le procès de plaisir antisocial fait à l'homosexualité masculine, car ces plaisirs n'aboutissent pas à la vie. Un autre libelle intitulé Le Courrier extraordinaire des fouteurs ecclésiastiques ou correspondance secrète et libertine associe le clergé à la débauche sexuelle et certains de ses passages concernent la sodomie masculine :

« Le plus grand bougre du monde, ah! C'est bien notre curé; et loin de foutre Raimonde, un garçon est préféré; La philosophie profonde dit à tous ah! Ce qu'il est; ne dérangez pas le monde, laissez chacun comme il est $»^{24}$.

(22) Le bordel apostolique [...], op. cit., p. 9.

(23) René RÉmOND, op. cit., p. 28-29.

(24) Le courrier extraordinaire des fouteurs ecclésiastiques, pièce révolutionnaire réimprimée textuellement sur l'édition originale publiée en 1790 et devenue très rare, précédée d'une notice bibliographique, Neuchâtel, impr. par les presses de la Société des bibliophiles cosmopolites, 1872 , p. 38. 
Un troisième pamphlet, sous le titre Étrennes aux fouteurs ou le calendrier des trois sexes, datant de 1793, associe également la pratique de la sodomie au clergé. Dans un passage de ce libelle, intitulé « La ressource du clergé », on peut lire : "Au surplus, si faute d'écus, tous nos soupirs sont superflus, faut-il qu'on se désole? Et con seul fait-il décharger? Non, le cul peut remplacer : c'est ce qui nous console $»^{25}$. Il faut noter que dans cet écrit la sodomie masculine n'est envisagée comme fantasme que parmi des débauches diverses, ce qui est parfois le cas dans ce type de libelles. Ceci est particulièrement visible dans un autre passage de ce pamphlet intitulé « Les cinq sens ou les trois générations $»^{26}$.

Enfin, parallèlement au clergé, certains pamphlets attaquent l'ancienne noblesse, l'ancienne Cour de France et les plus hauts personnages de l'État. Ces élites sont affublées de pratiques sexuelles sodomites pour montrer leur état de dépravation. Dans ce cas, la sodomie masculine n'est citée que parmi des débauches diverses qui donnent une image de plaisirs stériles et d'immoralité de ces anciennes élites. Dans Les délices de Coblentz ou anecdotes libertines des émigrés français, texte daté de 1792 , au fil des pages, des attaques sont portées contre d'anciens personnages importants de la Cour fuyant la France révolutionnaire. Le prince de Condé, défini comme l'âme de la noblesse française, y est qualifié de « pédéraste » et son hôtel est décrit comme le lieu de tous les plaisirs, festins, bals et autres parties nocturnes :

« Il se plaît à prodiguer aux plus basses prostituées, qu'il fait venir à grands frais pour assouvir les feux de sa lubricité. Sans cesse, il varie ses plaisirs. Fouteur et pédéraste, il s'échappe des bras des putains que pour enculer un jeune gentilhomme $»^{27}$.

Le plaisir de la sodomie masculine n'est ici que l'aboutissement de fantasmes débridés. Des anecdotes sur le futur Louis XVIII soulignent aussi son peu d'empressement auprès des femmes et, parallèlement, les rapports homosexuels qu'il aurait entretenus avec Condé. Occupé avec « sa dame de Balby qu'il ne sert pas mieux que son épouse $»^{28}$, « Stanislas Xavier [la] flagellait [...] pendant que Condé l'enculait. Ils se regardaient dans les glaces qui réfléchissaient parfaitement leurs attitudes lubriques et

(25) Étrennes aux fouteurs ou le calendrier des trois sexes, A Sodome et à Cythère et se trouvent plus qu'ailleurs dans la poche de ceux qui le condamnent, 1793, p. 49.

(26) Ibid., p. 7-9.

(27) Voir Les délices de Coblentz, Coblentz, Sn., 1792, p. 16-17 (BNf ENFER-1428).

(28) Ibid., p. 26. 
sodomites $»^{29}$. La sodomie est dans ce passage associée au sadisme. Une scène d'amour lesbien est aussi présente ${ }^{30}$. On découvre le thème de la prétendue déformation des organes génitaux lorsqu'ils sont utilisés pour des rapports homosexuels; ce thème se retrouvera plus tard dans la médecine légale. L'analyse des termes employés dans ce texte est parlante: l'âme de la noblesse française est associée à « ce réduit de tous les plaisirs ». La pratique de la sodomie est associée aux quartiers de noblesse : « six degrés de noblesse pour être enculé par ces princes ».

Un autre pamphlet, intitulé Le bordel patriotique institué par la reine des français pour les plaisirs des députés à la nouvelle législature, édité en 1791, met en scène la reine de France et les députés de l'Assemblée, pour lesquels elle instaure un bordel. On peut y lire des passages significatifs pour notre propos :

«Et quand vous serez rassasiés de l'une ou de l'autre, vous trouverez pour réveiller vos sens assoupis des ganymèdes modernes qui rallumeront vos feux amortis [...] Tous les suppôts de Priape, quel que soit leur caractère seront admis dans le bordel patriotique [...] Accourez, ribauds, fouteurs de tout genre, arrivez, maquerelles et putains de toutes les conditions, recevoir les instructions $»^{31}$; «Pour prévenir les désirs de tous les sacrificateurs, pour procurer du plaisir à tout le monde, elle n'a pas oublié de recevoir dans ses déduits amoureux, des ganymèdes, des bardaches, des pédérastes, des gamahucheurs, des gamahucheuses, des tribades, des sodomites, des enculeurs. Toutes les passions, tous les goûts des deux sexes y seront pleinement satisfaits; un homme y jouira avec un homme, et une femme avec une femme $[\ldots] »^{32}$.

Des personnages notables apparaissent au fil des pages, ainsi $\mathrm{La}$ Fayette qui avoue ses péchés passés :

« J'ai aussi donné dans le péché antiphysique, mais ce goût m'est passé. Je fous aujourd'hui en con, et non en cul. La pédérastie, la sodomie ne me tentent plus, j'aime mieux m'exposer à puiser à pomper la vérole dans le vagin des putains, que d'enculer un bougre $»^{33}$.

(29) Ibid., p. 44.

(30) Ibid., p. 54-55.

(31) Voir Le bordel patriotique institué par la reine des français pour les plaisirs des députés à la nouvelle législature, Aux Tuileries, chez les marchands d'ouvrages galans, 1791, p. 11 .

(32) Ibid., p. 12-13.

(33) Ibid., p. 39. 
Ainsi encore Bailly, qui fait l'apologie de l'homosexualité en évoquant les plus grands hommes de l'Antiquité et de Frédéric II.

« Je n'ai pas été plus sage que vous; mais les femmes n'ont pas excité mes plus vives passions. J'aime les hommes de préférences. Quoique je me sois marié tous comme un autre, par des considérations secrètes, j'aime mieux le derrière de ma femme que son devant, et pour vous parler vrai, je paie mon domestique le double que vous payez les vôtres, parce que j'ai le plaisir de l'enculer, et qu'il se prête commodément à ma fureur »; «Je suis le partisan des passions des plus grands hommes de l'Antiquité. Socrate, le sage Socrate était un fouteur en cul. Il n'a médit les cons que parce qu'il foutait en cul le jeune Alcibiade $»^{34}$.

Quant à Danton et Marat, ils s'apprêtent à avoir une relation homosexuelle : "Je vais vous donner une preuve d'attachement. Je vais vous enculer mais vous enculer de la bonne manière. Marat baisse la culotte et présente son cul $\aleph^{35}$. Ce pamphlet veut visiblement faire paraître les élites politiques comme dépravées et ne pensant qu'à leurs plaisirs purement hédonistes. En même temps la sodomie masculine est un plaisir distinct : les amateurs du cul qui se différencient des amateurs du con, mais on observe un passage d'un plaisir à un autre. Les fantasmes ne sont pas cloisonnés dans ce pamphlet. La sodomie masculine n'est pas encore détachée du concept plus général de débauche. Ce pamphlet s'explique aisément, car il fut rédigé en 1791. Les difficultés, les dangers, s'amoncellent sur la France révolutionnaire : difficultés financières et, le 20 juin 1791 , la fuite de la famille royale par Varennes. Au retour des souverains dans Paris, l'Assemblée constituante émit l'hypothèse que le souverain avait été enlevé. Du coup, il y eut divorce entre l'Assemblée qui, de fait, innocentait Louis XVI, et une partie de l'opinion qui le condamnait. Ces pamphlets relient ainsi la pratique de la sodomie entre hommes à la décadence d'anciennes élites. La pratique de la sodomie masculine est présentée, comme dans la littérature licencieuse d'Ancien Régime, comme la résultante de l'excès et de la dépravation sexuelle. L'homosexualité masculine fait partie, dans ces pamphlets, des plaisirs inutiles dans une époque de régénération et ils sont antisociaux. Parfois les pamphlets attaquent politiquement un acteur précis des événements révolutionnaires. Ainsi en est-il de Charles marquis de Villette, né à Paris en 1736, mort en 
1793, qui fut député de l'Oise à la Convention nationale et un sodomite notoire. Le pamphlet La vie privée et publique du ci-derrière marquis de Villette le présente comme un sodomite de toujours. Il aurait eu dès son plus jeune âge le goût des amours masculines : « il portait en lui-même le germe du vice affreux, qu'il affiche actuellement $»^{36}$; «On le trouva dans un chantier, par un froid excessif, et au milieu de la neige, enculant un petit décroteur $\gg{ }^{37}$. Dans le cas du marquis de Villette, le sodomite ne peut contenir ses pulsions sexuelles, et le plaisir de la sodomie se double de la transgression des barrières sociales. Dans le pamphlet, Les enfants de Sodome à l'Assemblée (1790), les homosexuels masculins sont présentés sous les traits d'une sorte de franc-maçonnerie, pratiquant le prosélytisme, et voulant imposer leur mode de vie. On assiste à une assemblée générale de l'ordre de la manchette ou des sodomites. Des personnages notoires sont là encore cités :

« Il sera mis sous presse et dans le plus court délai possible un manuscrit sauvé de l'embrasement de Sodome, ayant pour titre Traité élémentaire de l'Anti-physique [...] à l'usage des prétendants et des jeunes bardaches: quatre des plus anciens de l'ordre seront tenus d'en soigner l'impression; savoir, Barea de Girac, évêque de Rennes, Bourdelles, évêque de Soissons; le comte de Montrevel, maréchal-de-camp, et le marquis de Visé, lieutenant général des armées du roi ${ }^{38}$.

Ce pamphlet fait paraître plusieurs membres des élites comme adeptes de la sodomie. Il y a une atmosphère de monde inversé ou d'un ordre inversé. Ce texte possède un ton excessif dans sa manière de présenter l'homosexualité masculine parisienne, probablement dans le but de démontrer les progrès de la sodomie. D'autant plus que l'analyse lexicologique de ce texte laisse apparaître un emploi notable des qualificatifs " postérieurs », « culs », qui présentent l'homme adepte de ces plaisirs comme préoccupé uniquement par la pratique de la sodomie au sens pur.

Tous ces textes cités utilisent politiquement la sodomie masculine pour discréditer les anciennes élites, noblesse et clergé. Parfois, de nouvelles élites révolutionnaires sont également déconsidérées. La sodomie masculine semble servir de principale accusation pour mettre hors du jeu

(36) La vie privée et publique du ci-derrière marquis de Villette Lille, GCK, 1989, p. 2.

(37) Ibid., p. 5.

(38) Les enfants de Sodome à l'Assemblée nationale ou députation de l'ordre de la manchette, Lille, GCK, 1989, p. 25-26. 
social et politique celui que l'on vise. La sodomie masculine est aussi présentée comme incompatible avec le nouvel ordre social. On oppose ainsi à ces représentations, l'époque de régénérescence qui nécessite des élites en opposition avec ces descriptions. Pourquoi semble-t-on utiliser à ce point le ressort de la vie intime et de la sexualité? La Révolution française entre 1789 et 1794 n'a cessé d'étendre le domaine de la vie publique et cela va préparer le mouvement romantique du retour sur soi ${ }^{39}$. Ceci explique peut-être la façon d'utiliser la vie privée de manière si caractéristique que nous observons dans ces années? D'une manière générale, il y a un ton de moralisme très prononcé car ces textes décrivent ce que font d'anciennes élites, chassées parce que décadentes, dévergondées, et donc ce qu'un révolutionnaire ne doit pas faire. D'autre part, nous l'avons souligné, au cours du XVIII ${ }^{\mathrm{e}}$ siècle il y eut le développement d'une contre-société sodomite parisienne et certains grands seigneurs ou certains clercs y jouèrent un rôle. Par ailleurs, le fait de dénoncer les mœurs sodomites des anciennes élites n'est pas nouveau, à l'orée de la Révolution on en trouve déjà la trace dans des textes, tel que La foutromanie de Gabriel Sénac de Meilhan, texte datant de 1780. Précisément, dans ce texte, l'auteur attaque l'homosexualité qui va progresser et on peut y découvrir des propos assez violents contre le clergé. L'utilisation politique de la dépravation sexuelle et de l'homosexualité en particulier est le premier facteur observé dans l'analyse de cette littérature révolutionnaire. Elle démontre une volonté de rigorisme des mœurs : le révolutionnaire parfait se construit au moins idéologiquement en opposition à ce qui est décrit dans ces libelles. Il n'est pas dépravé et pratique la sexualité conjugale. Il fait preuve, de ce fait, d'altruisme, car les plaisirs des anciennes élites ne sont décrits que pour démontrer leur égoïsme face aux défis auxquels la France révolutionnaire doit faire face. L'intérêt de ces pamphlets révolutionnaires est de voir combien la sodomie masculine est construite comme un contre-modèle.

\section{La sodomie masculine comme plaisir antinaturel et hédoniste par essence}

D'autres images tout aussi, sinon encore plus, violemment négatives vis-à-vis de la pratique de l'homosexualité masculine apparaissent : elle est désignée comme un plaisir antinaturel que l'on oppose au « plaisir

(39) Voir Philippe ArıÈs, Georges Duby, Histoire de la vie privée, T 4, De la Révolution à la Grande guerre, Paris, Seuil, 1987, réed. 1999, p. 19. 
sain, naturel ». Plusieurs libelles de l'époque révolutionnaire définissent la sodomie masculine comme un plaisir antinaturel, répugnant, avec en parallèle la définition «d'un plaisir sain » conjugal. Ce type de discours pourrait signifier que l'on se trouve engagé dans un processus d'évolution qui conduira à opposer deux comportements sexuels jugés incompatibles. Cette opposition et cette vision transparaissent dans plusieurs écrits. Il y a là, par rapport au thème précédent, une volonté de définir et de circonscrire le plaisir homosexuel masculin. La Requête en faveur des putains, des fouteuses, des maquerelles et des branleuses contre les bougres, les bardaches et les brûleurs de paillasses, datée de l'an II, exalte la virilité et rabaisse la pratique de l'homosexualité masculine ${ }^{40}$ avec des images fortement répugnantes : « Je déteste les bardaches et les bougres qui pêchent les étrons à la ligne et lorsque je bandais, je me serais branlé jusqu'au sang, plutôt que d'insinuer mon vit entre des fesses dont je l'aurais tiré tout merdeux $\rangle^{41}$. La pratique de l'homosexualité masculine est mise ici en parallèle avec les excréments, avec un plaisir scatologique ${ }^{42}$. Il y a également une volonté de pénaliser à nouveau l'homosexualité en soi : "J'ai présenté à nos fouteurs de l'Assemblée une requête en votre faveur pour que tout bougre et bardache soit puni comme il le mérite $»^{43}$. On perçoit à travers ce type d'écrits combien la disparition du crime de sodomie n'allait pas de soi pour certains. Au fil de ce texte, on oppose le comportement du patriote viril, dit sain sexuellement, aux pratiques homosexuelles prêtées aux nobles, par une analyse qui met en avant l'homme tombé dans le péché et la colère des dieux ${ }^{44}$. Ce qui est une claire allusion à l'histoire biblique de Sodome. Le plaisir avec la femme semble incarner la régénération révolutionnaire et la sodomie masculine le contraire.

D'autres textes, sans parler directement de sodomie masculine, font l'éloge du bon comportement sexuel et, par contrecoup, un comportement sexuel marginal se trouve par contraste stigmatisé. Le texte intitulé Les fouteurs de bon goût à l'Assemblée nationale peut l'illustrer. Il fait l'éloge des beautés et du charme du sexe féminin, tandis que l'amour et la sexualité conjugale deviennent visiblement un service dû à la nation.

(40) Voir Requête en faveur des putains, des fouteuses, des macquerelles et des branleuses; contre les bougres, les bardaches et les bruleurs de paillasses, A Gamahuchon : et se trouve chez toutes les Fouteuses Nationales, L'an second de la régénération foutative.

(41) Ibid., p. 6.

(42) Ibid., p. 13, p. 16.

(43) Ibid., p. 7.

(44) Ibid., p. 13. 
Ceci fait de l'homme pratiquant l'homosexualité, un anti-patriote; c'est précisément ce qui peut expliquer autant le lien entre homosexualité et anciennes élites dans cette littérature. Un autre libelle, La confédération de la nature ou l'art de se reproduire, attribué à Alexandre-Jacques du Coudray (1744-1790), fait l'éloge de la sexualité conjugale en employant une langue très verte. Parallèlement, on peut y lire des propos violents sur la sodomie masculine. Certains passages opposent les deux comportements sexuels :

«Attendons pour baiser que la nature sage, ait entièrement conformé son ouvrage, il faut que chaque chose soit mise en son lieu, que la tête et le tronc répondent au milieu, que dans un seul fourreau les pièces assorties, ne forment qu'un seul tout des trois nobles parties. Et que vos deux couillons, trop lâches, s'éloignent, n'arrêtent le torrent du sperme jaillissant, évitons ces excès, laissons à l'Italie, des sales enculeurs, l'honteuse sodomie, le vit est pour le con, mais pour y parvenir le chemin est glissant et pénible à tenir $»^{45}$.

Ici, la sodomie masculine est assimilée à une forme de malpropreté et on insiste sur l'aspect contre-nature. D'autres textes font encore plus clairement la comparaison entre un comportement sexuel normal et un comportement jugé anormal et indigne d'un homme tel qu'il doit être. Dans Trois petits poèmes érotiques, de Gabriel Sénac de Meilhan, on peut lire : « Oui disait en baisant, vit ferme à va te con, l'anus est loin d'offrir tous les plaisirs du con, je reconnais que la sage nature, ne nous as fait des vits que pour cette ouverture $»^{46}$. Avec ce type de propos, la sodomie masculine apparaît comme un faux plaisir, tandis que le vrai plaisir est celui pris avec une femme, représentée par son sexe « le con ». De son côté, le libelle intitulé L'ode aux bougres décrit celui qui s'adonne à l'homosexualité justement comme un ennemi des femmes, donc de la procréation et de la vie : "Un amant qui parcourt une jeune beauté, goûte quand il la fait, une ivresse dans l'âme, qui va jusqu'au délire et qui fait qu'il se pâme, mais un bougre sent dans ses sales désirs, qu'un bonheur en idée, et que de faux plaisirs $\gg{ }^{47}$. Les descriptions des bardaches fort beaux et de leurs

(45) A. J. Du Courtray, La Confédération de la nature, ou l'Art de se reproduire, A Londres, i.e. [Paris?], 1790, p. 11-12.

(46) Gabriel Senac De Meillan, Trois petits poèmes érotiques, a Bâle, impr. exclusivement pour les membres de la Société des bibliophiles, les amis des lettres et des arts galants, sd., chant troisième p. 11.

(47) Ode aux bougres, sl., 1789, p. 4. 
visages peints des plus belles couleurs ${ }^{48}$ dessinent déjà un personnage passif féminin. Cette image préfigure l'inverti, représentation qui s'imposera dans la seconde moitié du XIX ${ }^{\mathrm{e}}$ siècle : "Monstres que la nature enfante parmi nous! Bardaches, bardachés, objet de mon courroux; exécrables pécheurs d'excréments à la ligne, dont je vois tous les jours croître la race indigne $[\ldots]$ bougre retirez vous, je vais chanter le con $»^{49}$. Dans ce texte, comme dans un précédent, la sodomie masculine est une fois encore liée à une image excrémentielle, voire scatologique.

Ainsi, les textes ici cités sont significatifs, car ils opposent deux comportements sexuels irréductiblement dissemblables. Ceci est d'autant plus intéressant qu'existait sous l'Ancien Régime une certaine habitude de bisexualité aristocratique. Comme le souligne l'universitaire Elizabeth Colwill, la bisexualité masculine des aristocrates qui disposent du corps d'autrui, est lue comme une dénonciation de leur omnipotence ${ }^{50}$. Par rapport à la sodomie masculine, cette opposition de deux comportements démontrerait que le comportement homosexuel masculin, comme globalement au XVIII ${ }^{\mathrm{e}}$ siècle, est bien l'objet d'un débat et qu'il commence à être perçu dans certains écrits comme un comportement sexuel particulier, opposé à un comportement normal. La sodomie masculine semble incarner le corps usé et flétri de l'aristocrate et la pratique de la sexualité avec une femme, le corps viril du patriote ${ }^{51}$. Le comportement homosexuel masculin est donc présenté à l'aune de certains de ces textes comme un comportement particulier, excluant de plus en plus la sexualité conjugale.

Parallèlement, et ceci semble étroitement lié à l'image de plaisir antinaturel, la sodomie masculine apparaît comme un plaisir purement hédoniste, égoïste, et lié à la décadence d'un monde ancien. Le fait d'attribuer ce comportement aux clercs qui sont célibataires et qui ne procréent pas est significatif, et l'attribuer à l'aristocratie d'Ancien Régime est encore une manière d'assimiler la pratique de l'homosexualité masculine à un comportement purement hédoniste. Ce comportement est imputé à une ancienne aristocratie qui vivait auparavant en parasite et ne s'occupait que de ses plaisirs égoïstes, donc l'homosexualité masculine est, parallè-

(48) Ibid., p. 6.

(49) Ibid., p. 3.

(50) Guillaume Mazeau dans son compte rendu de l'ouvrage de Dena Goodman (éd.), Marie-Antoinette, Writings on the Body of a Queen (New York, 2003), AHRF, 2003, vol. 334, $\mathrm{n}^{\circ} 1$, p. 185.

(51) Voir Patrick WALD-Lasowski, art. cité, p. 37. 
lement au fait d'être un plaisir antinaturel et fictif, un plaisir pur et donc égoïste par essence et non utile. Dans le libelle Le bordel apostolique, le lien entre le parasitisme supposé du clergé en matière économique et la pratique de l'homosexualité masculine est clairement établi. Des termes tels que « désirs antiphysiques » ou « ardeur de la bougrerie » voisinent avec « fruits de nos rapines », « secret d'être opulents ». La pratique de l'homosexualité masculine est mise en parallèle avec le désir d'argent facile. Il est nécessaire de mettre en parallèle ces représentations avec les mesures prises contre le célibat pendant la Révolution. Dès 1791, on établit une contribution annuelle mobilière, en partie commune à tous les habitants et en partie proportionnelle aux revenus. Des classes sont définies en fonction des revenus. En jouant sur ces classes qui définissent le montant de la contribution, on peut pénaliser arbitrairement ceux qui ne sont pas mariés. Les pères de plus de trois enfants sont placés dans une classe inférieure à celle qu'ils auraient dû occuper. En revanche, les célibataires seront placés dans une classe supérieure à celles où leur loyer les plaçait. Sous le Directoire, on surhausse le loyer des célibataires. En 1795, une contribution personnelle de cinq livres par an est demandée à tous les Français. On y ajoute une taxe somptuaire calculée, entre autres, sur le nombre de cheminées et l'on envisage aussi dans ce cas le problème des personnes seules. Des mesures militaires pénalisent les célibataires ${ }^{52}$.

\section{La sodomie masculine comme facteur de désordres}

Une dernière image se dégage de ces pamphlets, car l'homosexualité parait associée à des facteurs de désordres : prostitution, débauche de la jeunesse, maladies vénériennes. L'association avec la prostitution peut être mise au jour grâce à une analyse lexicale. Les termes "ribauds" ou «bordel» sont employés parallèlement aux termes «bougres» ou « bardaches ». Leur rapprochement associe clairement la pratique de la sodomie masculine à un pur plaisir qui, de fait, est associé à une forme de prostitution. De même, d'autres termes apparaissent dans ces textes, parallèlement à ceux désignant l'amour masculin : " réduits de tous les plaisirs », « putains »... faisant référence à un lieu de prostitution et mettant également en parallèle homosexualité masculine et racolage. Par exemple, Le Bordel patriotique institué par la reine des français pour les plaisirs des députés à la nouvelle législature a pour fond un bordel instauré par Marie-Antoinette pour les députés de l'Assemblée nationale.

(52) Jean-Claude Bologne, Histoire du célibat et des célibataires [...], op. cit., p. 196. 
Or, la pratique de l'homosexualité masculine intervient quand les autres plaisirs du sexe ont été épuisés. La sodomie masculine fait ici partie des débauches propres à des ambiances particulières, celles des bordels et, de plus, c'est en quelque sorte la partie la plus extrême de la débauche ${ }^{53}$. Quant au libelle intitulé Le courrier extraordinaire des fouteurs ecclésiastiques, il mêle la pratique de la sodomie masculine à d'autres débauches et toujours dans des lieux stigmatisés : « Il m'a fallu fréquenter les bordels et les chenils de la capitale, où grâce au ciel, je suis initié pour y recueillir les fragments de cette précieuse correspondance $»^{54}$.

Le lien entre sodomie masculine et débauche de la jeunesse est également établi. Des termes tels que « jeune garçon», "écolier», "petit satyre », " petit ganymède », " petit bardache » font référence à l'attirance pour les adolescents. Le pamphlet Les enfants de Sodome à l'Assemblée comporte sur ce point des passages qui font l'amalgame entre débauche de la jeunesse et sodomie masculine :

« Depuis on vit Monvel prendre en traitre, dans les Champs-Élysées, le pucelage de quelques écoliers et, forcé par la circonstance, aller en Bavière, donner des leçons publiques d'anti-physique $»^{55}$; "Le sieur Remy, ci-devant sergent d'armes, au régiment des gardes-françaises. Ce digne soutien de la manchette est généralement connu, pour avoir débauché la majeure partie des jeunes gens de sa compagnie $»^{56} ;$ "On vit Perducas, procureur au Châtelet de Paris, qui, parlant par révérence, est un des plus fermes appuis de cet ordre, tenir chez lui un bureau d'adresses, et indiquer aux jeunes novices initiés dans les mystères de l'anus $»^{57}$.

Il y a dans cette remarque l'idée que la sodomie masculine est un plaisir qui tend à corrompre des jeunes innocents. Le libelle Les petits bougres au manège comporte lui aussi des amalgames entre sodomie masculine et débauche des plus jeunes: " Je n'ai point l'ambition de monter le fougueux Pégase; et pourvu que j'enfourche un jeune blondin aux fesses rebondies; pourvu que je lui lave l'anus d'un déluge de foutre $»^{58}$.

(53) Le bordel patriotique [...], op. cit., p. 11.

(54) Le courrier extraordinaire des fouteurs ecclésiastiques : pièce révolutionnaire réimprimée textuellement sur l'édition originale publiée en 1790, Neuchâtel, Presses de la société des bibliophiles cosmopolites, 1872, p. 3.

(55) Les enfants de Sodome à l'Assemblée [...], op. cit., p. 4.

(56) Ibid., p. 45-46.

(57) Ibid., p. 6.

(58) Les petits bougres au Manège : l'an second du rêve de la liberté, Lille, GCK, 1989, p. $1-2$. 
Cette phrase semble décrire un type de relations sexuelles propre à une société d'ordres dans laquelle le petit «giton» ou le petit «satyre» est sous la dépendance d'un plus important et est toujours passif avec ce dernier. C'est le bougre qui débauche des jeunes gens ${ }^{59}$.

Enfin, plusieurs de ces sources font mention de la cristalline et plus globalement des maladies vénériennes. Par exemple, Le bordel patriotique instauré par la reine des français pour les plaisirs des députés affirme : «Frédéric II, roi de Prusse, si célèbre par sa valeur, et son génie n'aimait pas les femmes et se faisait enculer; témoin Baculard d'Arnaud qu'il appelait son berger et avec qui il gagna la cristalline $»^{60}$. Dans les Enfants de Sodome à l'Assemblée on peut également lire : « Tous médecins, chirurgiens, déclarés ou non, assassins par brevet de la faculté seront tenus de prêter leur ministère à la guérison de la cristalline $»^{61}$. De même, un peu avant le déclenchement des événements révolutionnaires, $\mathrm{La}$ foutromanie, libelle datant de 1780, comprenait déjà des passages caractéristiques à ce sujet : " Les monsignores imprudentes vermines dont Rome abonde, prestolets, lâches gitons, fouteurs à bas violets, de la vérole et de la cristalline $[\ldots] »^{62}$.

Ainsi, la sodomie masculine est assimilée à des excès vénériens, à la débauche. Dans beaucoup de ces pamphlets, elle n'est que la phase la plus extrême des plaisirs : le plaisir lorsque l'on a tout épuisé, comme dans le pamphlet Le bordel patriotique. Cependant, nous pouvons remarquer une tendance à opposer deux choix sexuels symbolisés par le con (sexe féminin) et le cul qui symbolise la sodomie. La tendance était déjà perceptible dans une littérature licencieuse de la fin du XVIII ${ }^{\mathrm{e}}$ siècle. Cette opposition apparaît dans ces libelles avec nettement plus de clarté, et ceci semble nouveau. Le fait d'opposer avec une telle clarté le comportement homosexuel, antinaturel et exclusif, au comportement « conjugal», sain et productif socialement, paraît novateur, de même que de faire allusion au fait démographique. Dans certains pamphlets, on décrit même des comportements et des attitudes caractérisant les pratiquants de la sodomie masculine. Il y a dans ce cas, une volonté d'identification. La teneur

(59) Voir Thierry Pastorello, Sodome à Paris : protohistoire de l'homosexualité masculine fin XVIII ${ }^{e}$ - première partie XIX ${ }^{e}$ siècle, thèse soutenue à Paris VII, 2009, p. 37-41.

(60) Le bordel patriotique [...], op. cit., p. 40.

(61) Les enfants de Sodome à l'assemblée [...], op. cit., p. 25.

(62) Gabriel Senac de Meilhan, La Foutromanie : poème lubrique, A Sardanapalis, aux dépens des amateurs. 1780, p. 65-66. 
de ces pamphlets doit être replacée dans le cadre de l'idée de régénération, idée forte à l'époque de la Révolution. Ainsi, le passé comportait un soupçon de corruption. On retrouve l'idée rousseauiste selon laquelle la rupture révolutionnaire ouvrait la voie à la régénération. Un peuple régénéré, notamment dans ses mœurs, ne se pervertit pas. Aussi retrouve-t-on dans ces pamphlets, en arrière-plan, une telle volonté de régénération des mœurs.

Thierry Pastorello

Docteur en histoire, Université Paris Diderot - Paris VII Laboratoire ICT Bibliothèque nationale de France 11 rue de l'Évangile Paris 75018 thierry.pastorello@cegetel.net 